\title{
Dihydromyricetin induces apoptosis and inhibits proliferation in hepatocellular carcinoma cells
}

\author{
JIE LIU $^{1 *}$, YANG SHU $^{1 *}$, QINGYU ZHANG ${ }^{1}$, BIN LIU $^{1}$, JUAN XIA $^{1}$, MINGNING QIU $^{2}$, HUILAI MIAO $^{1}$, \\ MINGYI LI ${ }^{1}$ and RUNZHI ZHU ${ }^{1}$ \\ ${ }^{1}$ Zhanjiang Key Laboratory of Hepatobiliary Diseases; ${ }^{2}$ Department of Urology Surgery, \\ Affiliated Hospital of Guangdong Medical College, Zhanjiang, Gunagdong 524001, P.R. China
}

Received October 31, 2013; Accepted June 5, 2014

DOI: $10.3892 / 01.2014 .2330$

\begin{abstract}
Hepatocellular carcinoma (HCC) is a life-threatening disease that is known to exhibit a poor prognosis. Therefore, it is important to identify an effective drug therapy for the treatment of HCC. Dihydromyricetin (DHM) is a flavonoid compound, isolated from the classical Chinese herb Ampelopsis grossedentata, which exhibits multiple pharmacological activities, including anticancer effects. In this study, the anticancer effect of DHM was investigated in nine different types of HCC cell lines via cell proliferation and immunoassays, as well as apoptosis detection. Two immortalized normal human liver cell lines were utilized to determine hepatotoxicity. The results revealed that DHM significantly inhibited cell proliferation and induced cell apoptosis in the HCC cell lines. However, DHM exhibited no cytotoxicity to normal human hepatic cell lines. Furthermore, it was found that DHM induced cell apoptosis in a p53-dependent manner. DHM upregulated p53 expression, and the upregulation of p53 increased the levels of the cleaved caspase- 3 protein, directly inducing cell apoptosis. These results indicate that DHM is a promising candidate for the treatment of HCC.
\end{abstract}

\section{Introduction}

Hepatocellular carcinoma (HCC) exhibits one of the highest incidences of morbidity and mortality worldwide (1), and presents the predominant histological subtype of primary liver cancer (2). As postsurgical recurrence of HCC is frequent and

Correspondence to: Dr Mingyi Li or Dr Runzhi Zhu, Zhanjiang Key Laboratory of Hepatobiliary Diseases, Affiliated Hospital of Guangdong Medical College, 57 South Renmin Road, Zhanjiang, Guangdong 524001, P.R. China

E-mail: hepatolab@163.com; hepatolab@gmail.com

E-mail: zhurunzhi@gmail.com

*Contributed equally

Key words: dihydromyricetin, hepatocellular carcinoma, p53, apoptosis, caspase-3 often fatal, surgery and liver transplant offer limited treatment options for HCC $(3,4)$. Consequently, it is important to identify an effective drug therapy for the treatment of HCC. The anticancer ability of certain traditional Chinese medicines has been accepted in cancer therapy (4).

Dihydromyricetin (DHM) is a type of flavonoid extracted from Ampelopsis grossedentata (Fig. 1), which exhibits pharmacodynamic effects, including scavenging of free radicals, anti-oxidative, antithrombotic and anti-inflammatory effects (5-7). In addition, DHM has been found to exhibit anti-alcoholic and anti-lipid peroxidation effects $(8,9)$. Previous studies have suggested that DHM exhibits a protective ability on the liver. In this study, the suppression of proliferation and induction of apoptosis by DHM was investigated in hepatocellular carcinoma cell lines.

\section{Materials and methods}

Main reagents. DHM (Sigma-Aldrich, St. Louis, MO, USA) was dissolved in dimethylsulfoxide (DMSO) at a concentration of $50 \mathrm{mM}$, and diluted to a working concentration using culture medium, prior to use. 3-(4,5-dimethylthiazol-2-yl)-2,5-diphenyltetrazolium bromide (MTT) and propidium iodide (PI) were also purchased from Sigma-Aldrich. RNase A was purchased from Thermo Fisher Scientific (Rockford, Waltham, MA, USA). The In Situ Cell Death Detection kit, POD was purchased from Roche (Basel, Switzerland), and the fluorescein isothiocyanate (FITC)-Annexin V staining kit was purchased from BD Biosciences (Franklin Lakes, NJ, USA). Monoclonal rabbit anti-human primary antibodies against Bax, Bak, p53, caspase-3, Caspase- 9 and $\beta$-actin were obtained from Cell Signaling Technology, Inc. (Boston, MA, USA). The polyclonal goat anti-rabbit secondary antibody was purchased from EarthOx Life Science (Millbrae, CA, USA).

Cell lines and cell culture. Human hepatocellular carcinoma cell lines (HepG2, QGY7701, QGY7703, QSG7701, Huh-7, MHcc97L, MHcc97H and SK-HEP-1), one mouse hepatocellular carcinoma Hepal-6 cell line and immortalized normal human liver HL7702 and L-02 cell lines were used in this study. HepG2, QGY7701, Hepal-6 and QGY7703 cell lines were obtained from Shanghai Maternal and Child Health Hospital (Shanghai, China). L-02, MHcc97L, MHcc97H, 
QSG7701, Huh-7 and SK-HEP-1 cell lines were purchased from the Shanghai Cell Bank of Chinese Academy of Science (Shanghai, China). The HL7702 cell line was obtained from the Chinese Academy of Science (Kunming, China). HepG2, QSG7701, L-02, HL7702 and Hepal-6 cells were maintained in RPMI-1640 medium (Gibco-BRL, Grand Island, NY, USA) supplemented with $10 \%$ fetal bovine serum (FBS; Gibco-BRL). QGY7701, QGY7703, Huh-7, SK-HEP-1, MHcc97L and MHcc97H cells were maintained in Dulbecco's modified Eagle's medium (Gibco-BRL) supplemented with 10\% FBS All cell lines were cultured at $37^{\circ} \mathrm{C}$ in a humidified incubator with an atmosphere of $5 \% \mathrm{CO}_{2}$.

Cell proliferation assay. The effects of DHM treatment on the cell proliferation of hepatic cancer cell lines was detected by MTT assay. Cells were seeded in 96-well plates at a density of $1 \times 10^{4}$ cells/well with $100 \mu 1$ culture medium. Following $24 \mathrm{~h}$, culture medium was removed and the same volume of medium, containing various concentrations of DHM, was added, respectively. Cells cultured in medium containing DMSO were used as a vehicle control. Following various periods of time, $5 \mathrm{mg} / \mathrm{ml}$ MTT solution was added to each group $(20 \mu 1 /$ well $)$, and then incubated at $37^{\circ} \mathrm{C}$ for $4 \mathrm{~h}$. Next, the liquid in each well was removed and replaced with $150 \mu 1$ DMSO. The absorbance was then detected using a microplate reader (PerkinElmer, Waltham, MA, USA) at a wavelength of $570 \mathrm{~nm}$ and the percentages of viable cells were compared with the control. The experiments were performed independently and at least in triplicate.

Cell cycle assay. Cells were plated onto $60-\mathrm{mm}$ dishes at a density of $3 \times 10^{6} \mathrm{cells} / \mathrm{dish}$. Following overnight growth, the cells were exposed to various concentrations of DHM, and then harvested when significant proliferation inhibition was observed. Cells were fixed in $70 \%$ ethanol water at $4^{\circ} \mathrm{C}$ overnight, followed by incubation with $100 \mu \mathrm{g} / \mathrm{ml}$ PI and $100 \mu \mathrm{g} / \mathrm{ml}$ RNase A in PBS at $37^{\circ} \mathrm{C}$ for $1 \mathrm{~h}$. DNA content was then determined by flow cytometry for the cell cycle distribution assay. The experiments were performed independently and at least in triplicate.

Annexin V staining. Annexin V-FITC/PI staining was used to detect apoptosis induced by DHM according to the manufacturer's instructions. Cells were cultured in six-well plates at a density of $1 \times 10^{5}$ cells/well. Following overnight growth, cells were treated with various DHM concentrations and harvested for the apoptosis assay. Untreated cells were used as a negative control. The experiments were performed independently and in triplicate.

Terminal deoxynucleotidyl transferase dUTP nick end labeling (TUNEL) assay. The late-stage apoptosis of hepatic cell lines was detected using the In Situ Cell Death Detection kit, POD (Roche). Cells were seeded in 96-well plates and, following DHM treatment, cells were fixed with $4 \%$ paraformaldehyde according to the manufacturer's instructions. The cells were then counterstained with 4',6-diamidino-2-phenylindole for $5 \mathrm{~min}$ at room temperature in the dark and observed under a fluorescence microscope (Olympus IX70; Olympus Corporation, Tokyo, Japan) to detect the apoptotic cells (TUNEL-positive cells).<smiles>O=C1c2c(O)cc(O)cc2O[C@H](c2cc(O)c(O)c(O)c2)[C@@H]1O</smiles>

Figure 1. Chemical structure of dihydromyricetin.

Western blot analysis. The expression of the apoptosis-associated proteins Bak, Bax, p53, caspase-3 and -9 were detected in hepatic cancer cell lines. Cells were suspended in lysis buffer on ice for $30 \mathrm{~min}$ and the cell lysates were cleared by centrifugation at $13,000 \mathrm{x} \mathrm{g}$ at $4^{\circ} \mathrm{C}$ for $10 \mathrm{~min}$. The supernatants were then collected and detected by bicinchoninic assay. Next, the cellular lysates containing equal amounts of total protein were separated by SDS-PAGE and transferred to polyvinylidene difluoride membranes. Membranes were then blocked using 5\% non-fat milk in Tris-buffered saline and Tween 20 (TBST) at room temperature for $1 \mathrm{~h}$, and the membranes were incubated at $4{ }^{\circ} \mathrm{C}$ overnight with the primary antibodies. The membranes were washed three times with TBST for 5 min each and incubated for $1 \mathrm{~h}$ with horseradish peroxidase-conjugated secondary antibodies. The bands were then analyzed by an enhanced chemiluminescence blotting detection system (FluorChem E; Proteinsimple, Santa Clara, CA, USA).

Statistical analysis. The data were obtained from at least three independent experiments and all results are presented as the mean \pm standard deviation. The differences between the groups were assessed using Student's t-test. Comparisons were relative to untreated controls. $\mathrm{P}<0.05, \mathrm{P}<0.01$ and $\mathrm{P}<0.001$ were considered to indicate a statistically significant difference.

\section{Results}

DHM inhibits the proliferation of HCC cell lines. MTT assay was used to investigate the potential inhibition of cell growth in HCC cells. As shown in Fig. 2, in HCC cells treated with various concentrations of DHM for 24,48 and $72 \mathrm{~h}$, cell viability was significantly inhibited in a dose- and time-dependent manner. Following $24 \mathrm{~h}$ of treatment with $100 \mu \mathrm{M}$ DHM, the proliferation of HepG2, QSG7701, QGY7701 and Hepal-6 cells was significantly inhibited $(\mathrm{P}<0.01)$. Furthermore, following $48 \mathrm{~h}$ of treatment with $50 \mu \mathrm{M}$ DHM, the proliferation of HepG2, Huh7, QSG7701, QGY7701 and Hepal-6 cells was markedly suppressed $(\mathrm{P}<0.001)$. The results showed that the inhibitory rate of MHcc97H, QGY7703 and SK-HEP-1 cells was significantly higher than the control group $(0 \mu \mathrm{M})$ following $48 \mathrm{~h}$ of treatment with $50 \mu \mathrm{M}$ DHM $(\mathrm{P}<0.05)$, while MHcc97L cells were significantly inhibited following treatment with $25 \mu \mathrm{M}$ DHM for $72 \mathrm{~h}(\mathrm{P}<0.01)$. Since the results revealed that the inhibitory rates were $<50 \%$ following treatment with $150 \mu \mathrm{M}$ DHM for $72 \mathrm{~h}$, these cell lines were considered to be less sensitive to DHM administration. Notably, DHM did not affect the cell growth of immortalized normal human liver HL7702 and L-02 cell lines. 

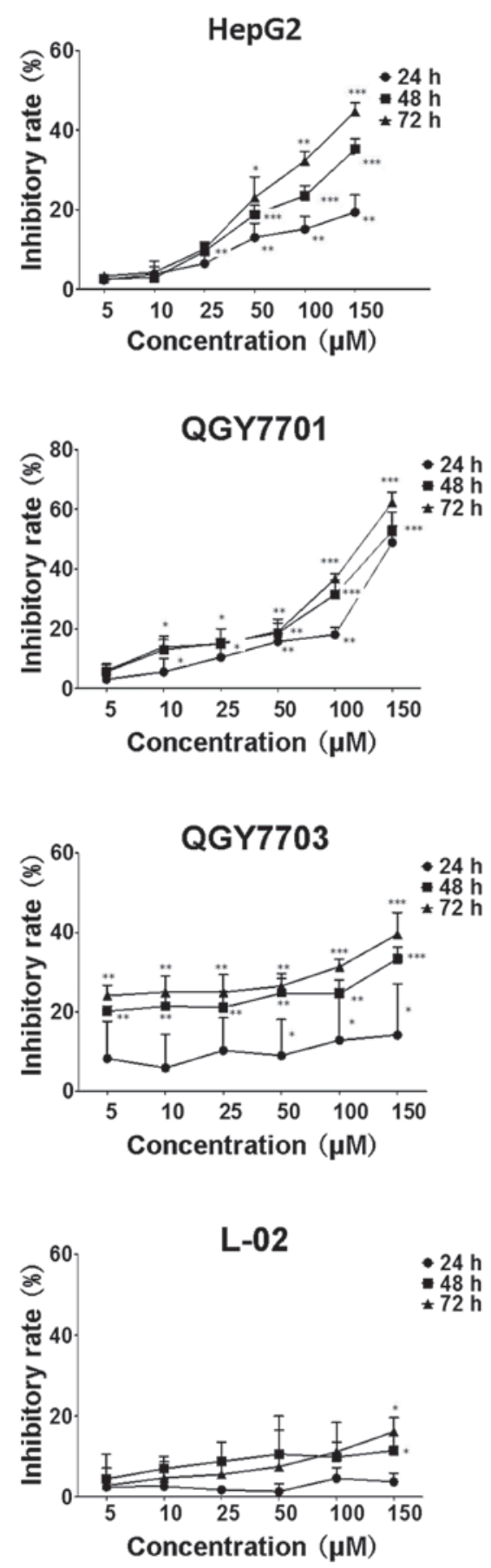
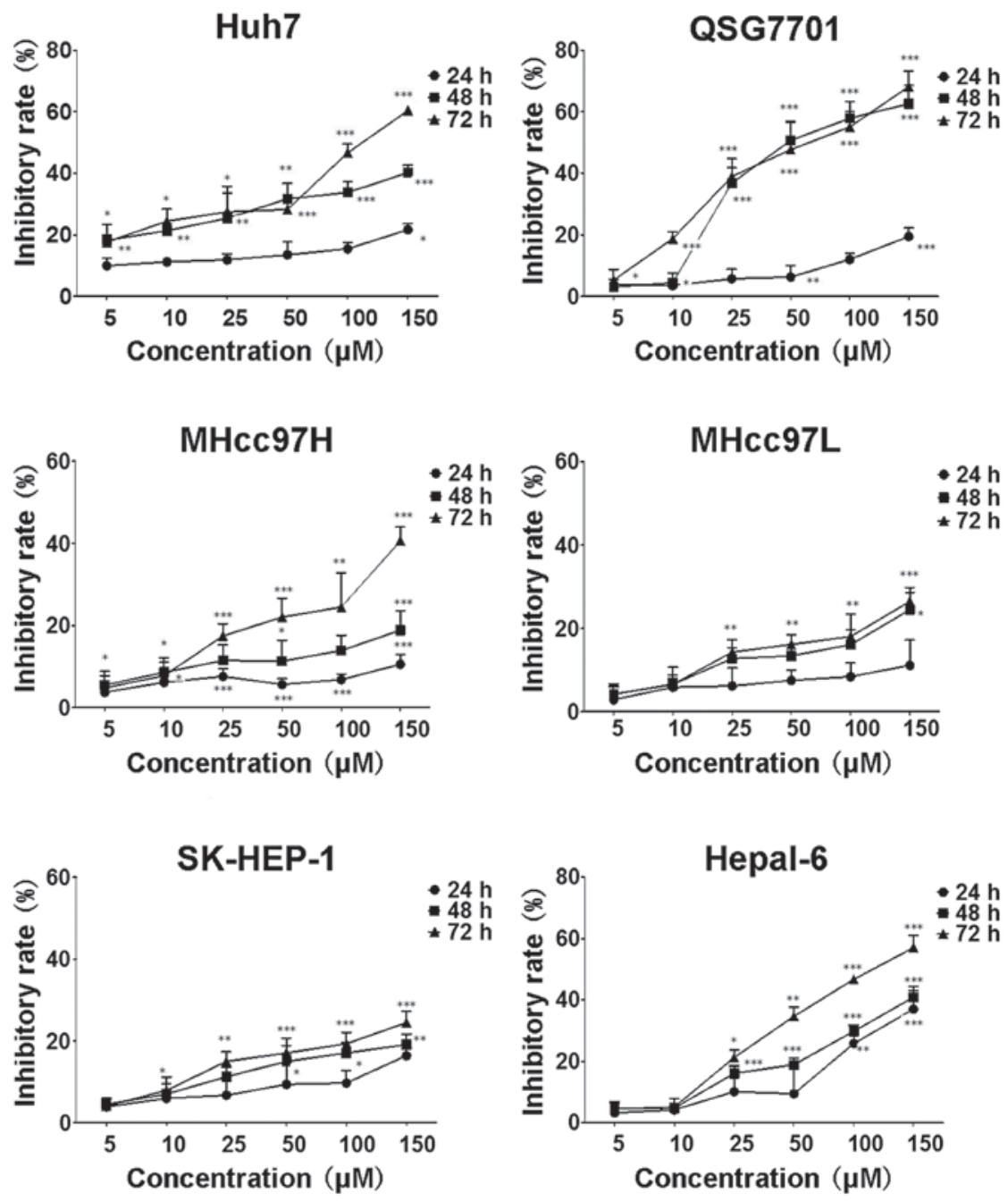

Figure 2. Cell growth inhibition rate of eleven different types of liver cell lines following DHM treatment. Cells were exposed to various DHM concentrations $(5,10,25,50,100$ and $150 \mu \mathrm{M})$ for 24,48 and $72 \mathrm{~h}$, and the inhibition rate of cells without DHM treatment was defined as 0 . Each sample was duplicated, and the figures present three independent assays $(n=4)$. Values are presented as the mean \pm standard deviation for at least three independent experiments performed in triplicate. ${ }^{*} \mathrm{P}<0.05,{ }^{* *} \mathrm{P}<0.01$ and $^{* * *} \mathrm{P}<0.001$, compared with the untreated $(0 \mu \mathrm{m})$ control. DHM, Dihydromyricetin.

DHM does not induce cell cycle arrest in HCC cell lines. The cell cycle arrest of HepG2, QGY7701, MHcc97L, Hepal-6 and HL7702 was detected by flow cytometry. No significant differences were identified between DHM-treated groups and the control (Fig. 3).

DHM promotes apoptosis in HCC cells. Flow cytometry was used to detect the apoptosis of HCC cells following DHM treatment. In the present study, HepG2 and QGY7701 cells were sensitive to DHM treatment. MHcc97L cells were insensitive to DHM treatment when compared with HepG2 and QGY7701 cells. Hepal-6 is a mouse hepatoma carcinoma cell line, in which a higher apoptotic rate may be induced than in human HCC cell lines. HL7702 is an immortalized normal human hepatocyte cell line that was used as the control. Apoptosis was detected in all cell lines by Annexin V staining following treatment with $\operatorname{DHM}(0,50$ and $100 \mu \mathrm{M})$ for 24 and $48 \mathrm{~h}$. The results revealed that following $24 \mathrm{~h}$ of treatment with DHM, apoptosis was induced in HepG2 and QGY7701 cell lines. DHM treatment for $48 \mathrm{~h}$ induced very 

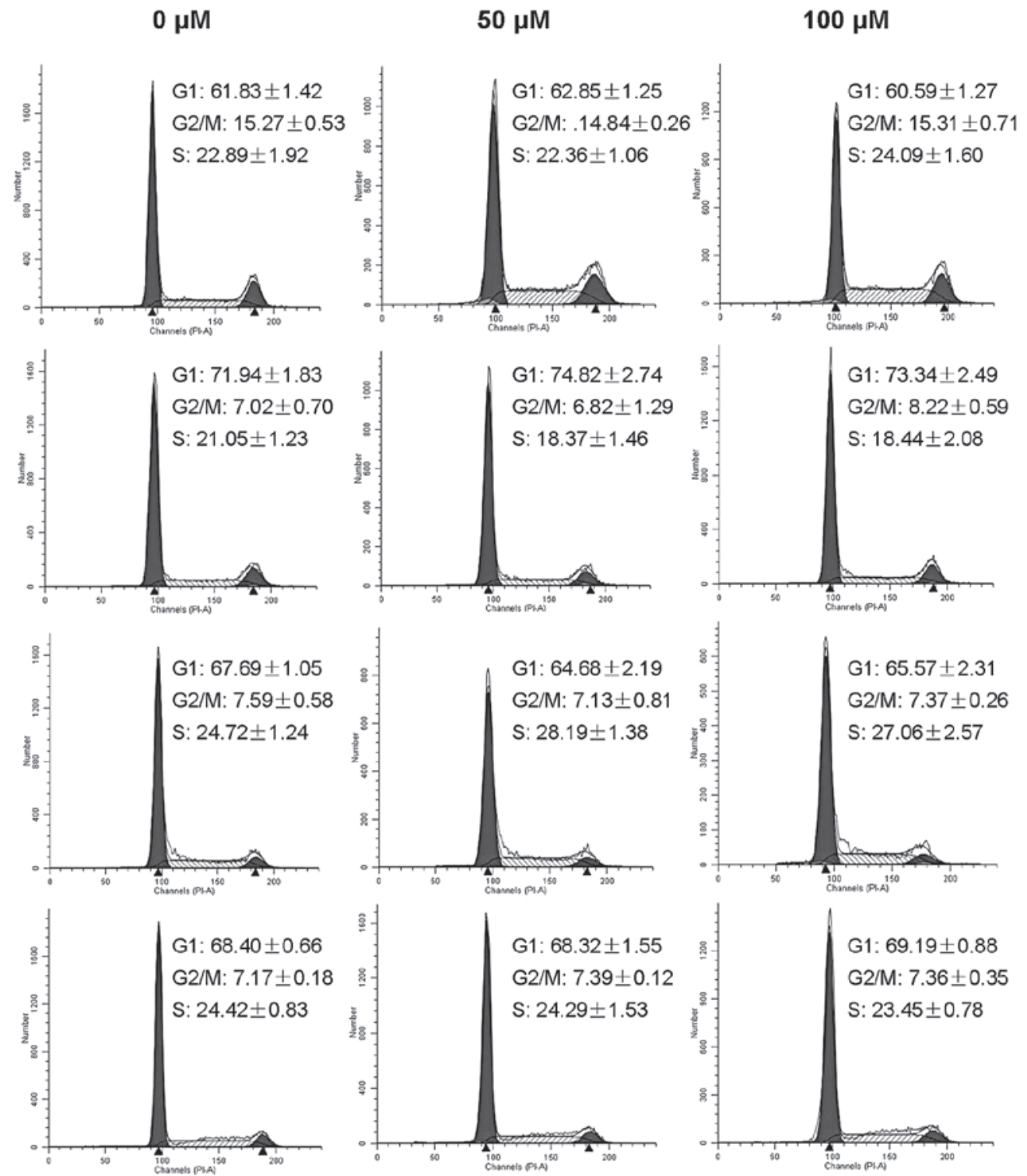

QGY7701 (24 h)

HepG2 (24 h)

QGY7701 (24 h)

MHcc97L (48 h)
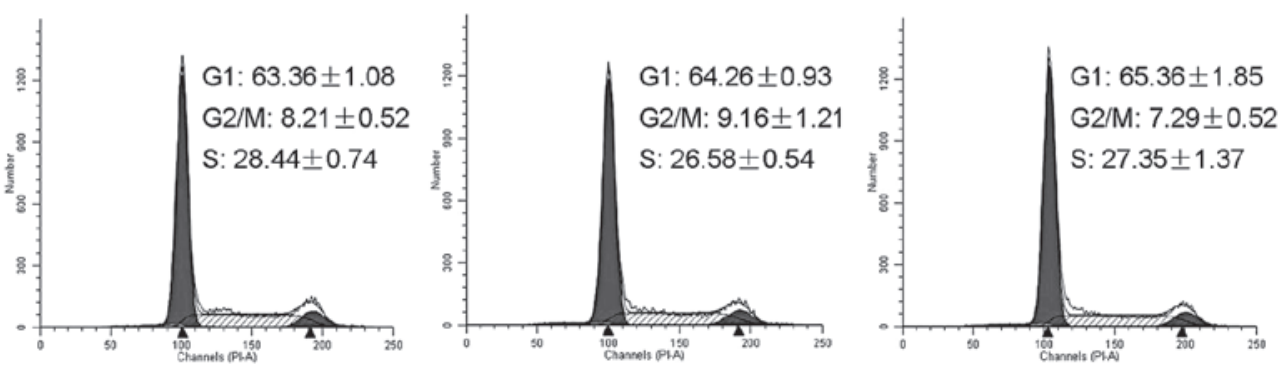

Hepal-6 (48 h)

HL7702 (48 h)

Figure 3. DHM does not induce cell cycle arrest. Cells were treated with various DHM concentrations $(0,50$ and $100 \mu \mathrm{M})$ for $24 \mathrm{~h}$ (HepG2 and QGY7701) or $48 \mathrm{~h}$ (MHcc97L, Hepal-6 and HL7702) and analyzed by flow cytometry. DHM, dihydromyricetin.

high levels of apoptosis in Hepal-6 cells. However, apoptosis was not observed in MHcc97L and HL7702 cells following $48 \mathrm{~h}$ of DHM treatment. Notably, the apoptosis rate significantly decreased in the HL7702 cell line following treatment with $50 \mu \mathrm{M}$ DHM, which indicated that lower concentrations of DHM exhibit protective effects on normal liver cells (Fig. 4A and B).

A TUNEL assay was used to detect the late-stage apoptosis of the cell lines following DHM treatment. Late-stage apoptotic cells were detected in the HepG2 and QGY7701 cell lines following treatment with DHM for $24 \mathrm{~h}$, and in the remaining cell lines following treatment with DHM for $48 \mathrm{~h}$. These results were consistent with the flow cytometry results (Fig. 4C and D).

DHM induces cell apoptosis via the activation of caspase-3 and subsequent upregulation of p53, Bax and Bak. In this study, western blot analysis was used to detect the expression of apoptosis-associated proteins in the QGY7701, HepG2, MHcc97L, Hepal-6 and HL7702 cell lines. The 
A

\section{HepG2 (24 h) QGY7701 (24 h) MHcc97L (48 h) Hepal-6 (48 h) HL7702 (48 h)}
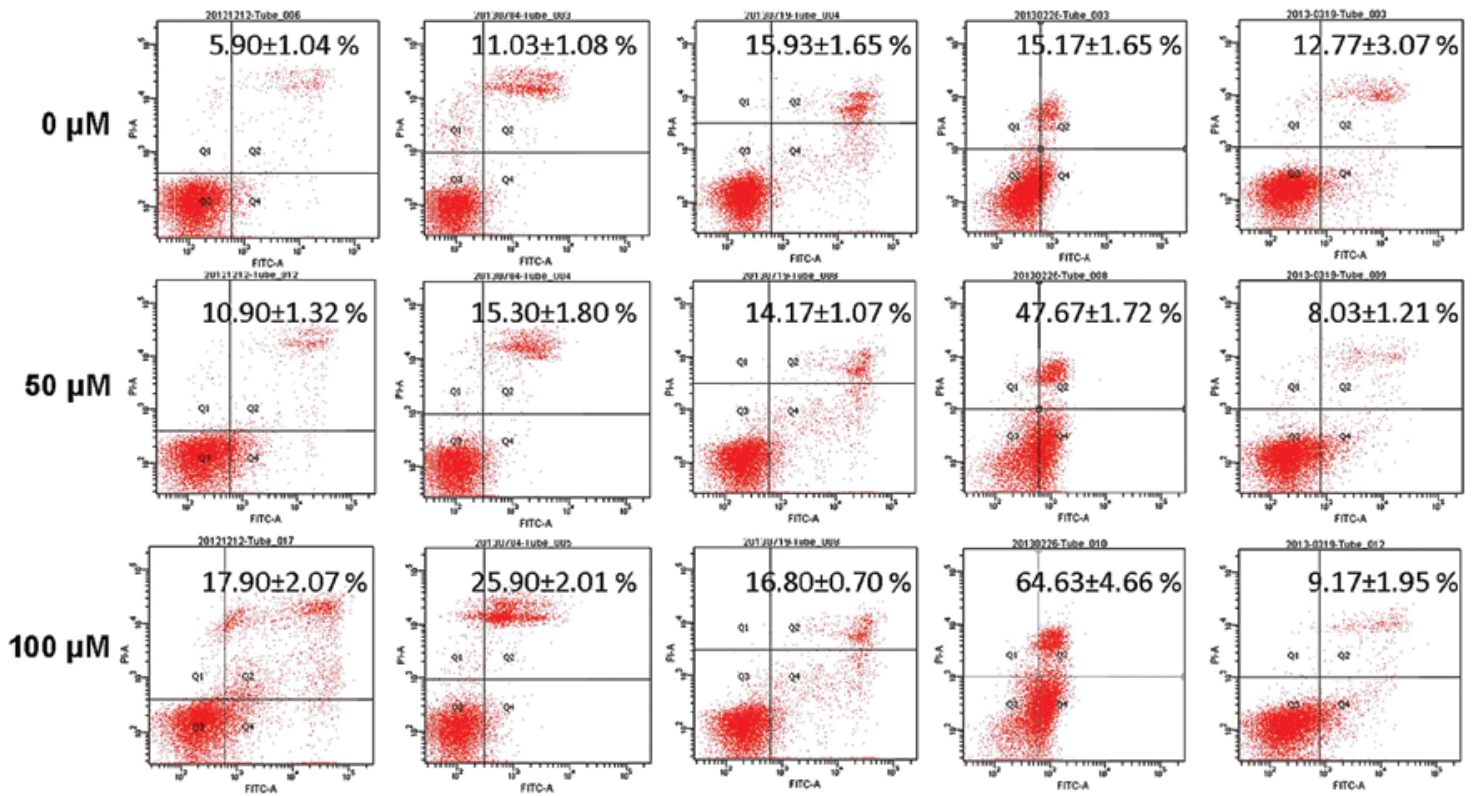

B

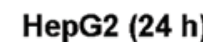

QGY7701 (24 h)

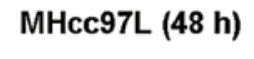

Hepal-6 (48 h)

HL7702 (48 h)
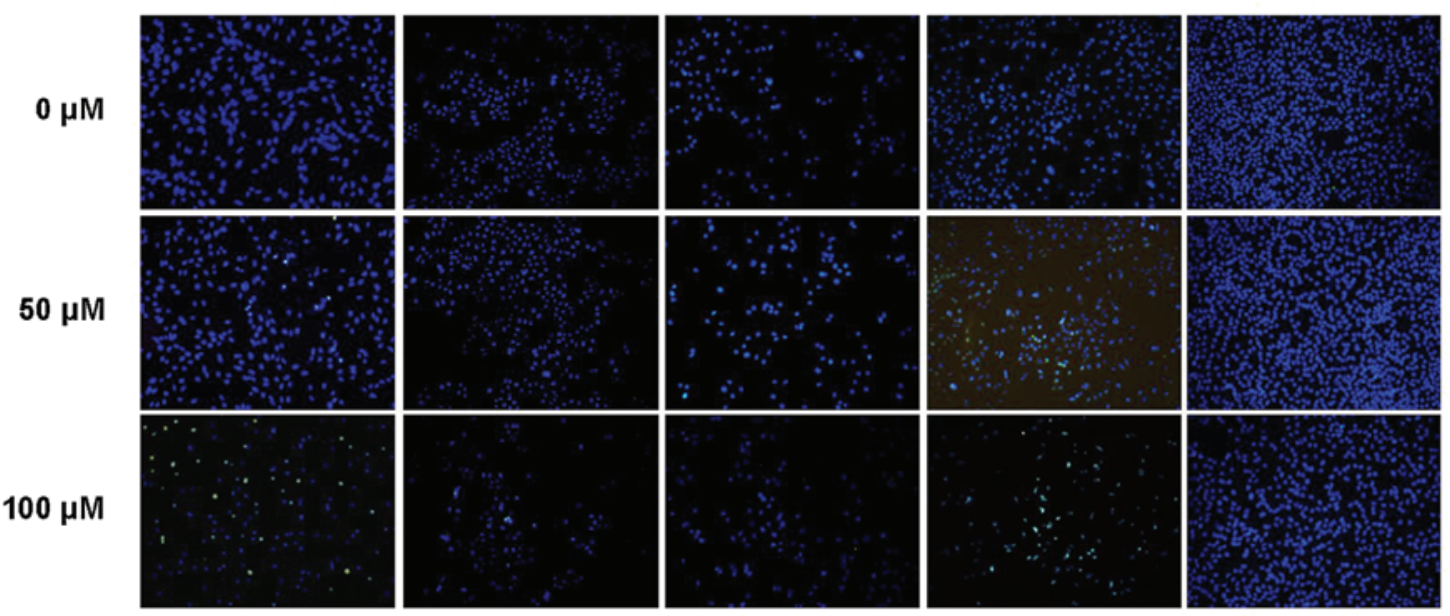

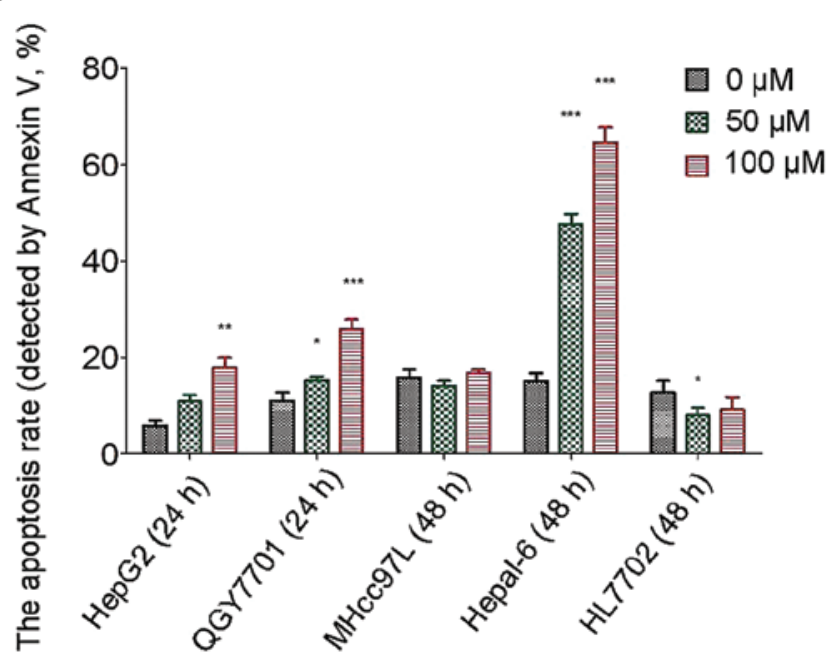

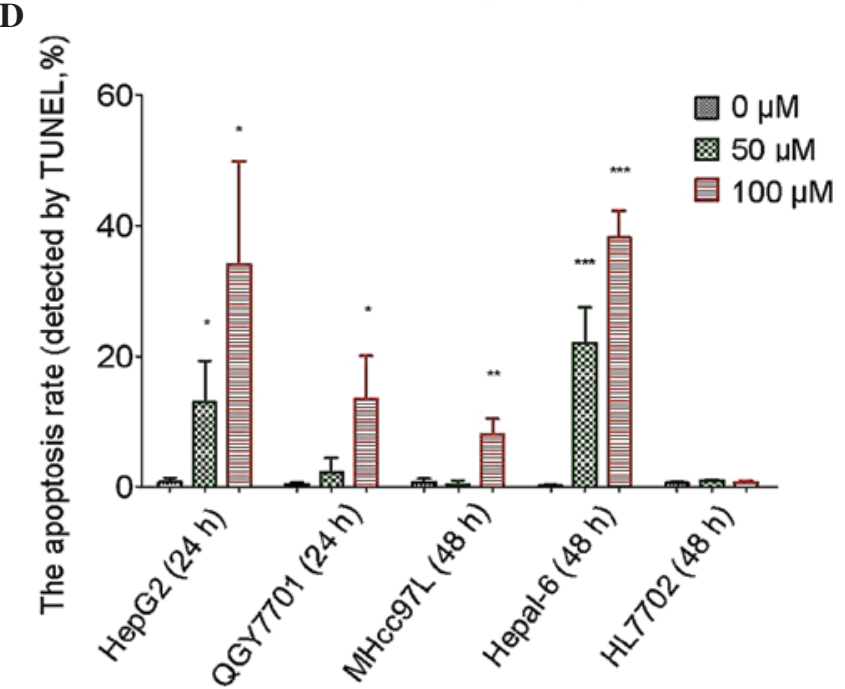

Figure 4. Flow cytometry revealed that DHM induces cell apoptosis. (A and B) HepG2 and QGY7701 cells were treated with various DHM concentrations (0, 50 and $100 \mu \mathrm{M})$ for $24 \mathrm{~h}$, and MHcc97L, Hepal-6 and HL7702 cells were treated with various DHM concentrations $(0,50$ and $100 \mu \mathrm{M})$ for $48 \mathrm{~h}$, and analyzed using the Annexin V staining method. (C and D) Terminal deoxynucleotidyl transferase dUTP nick end labeling assay was used to analyze the number of late-stage apoptotic cells following DHM treatment. The experiments were independently performed in triplicate. ${ }^{*} \mathrm{P}<0.05,{ }^{* * *} \mathrm{P}<0.01$ and ${ }^{* * * *} \mathrm{P}<0.001$, compared with the untreated $(0 \mu \mathrm{m})$ control. DHM, dihydromyricetin. 


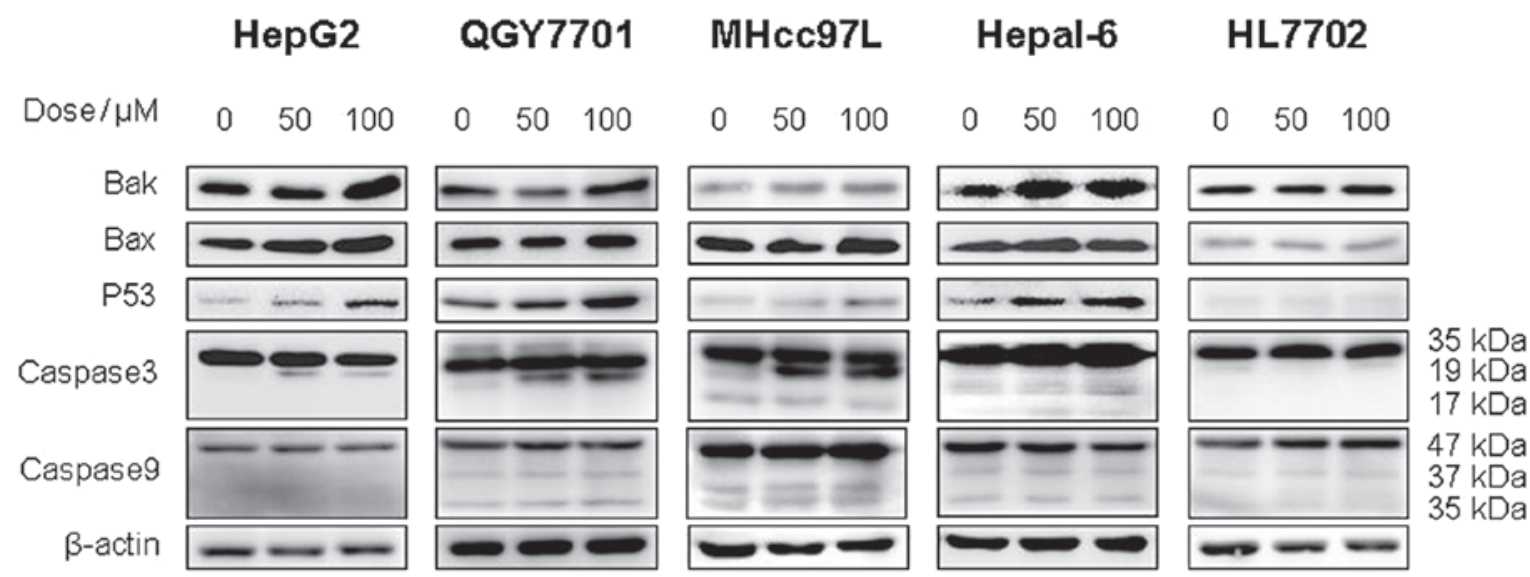

Figure 5. DHM induces cell apoptosis of HCC possibly via the p53/Bax and caspase-3 signaling pathways. (A) QGY7701, (B) HepG2, (C) MHcc97L, (D) Hepl-6 and (E) HL7702 cells were treated with DHM, and the levels of p53, Bax, Bak, caspase-3 and -9 proteins were detected by western blot analysis. DHM upregulated p53, then $\mathrm{p} 53$ recruited the activated form of caspase-3, which induced cell apoptosis of HCC in a concentration-dependent manner. No significant differences in the apoptosis-associated proteins (p53 and cleaved caspase-3) were identified following DHM treatment of the normal hepatic HL7702 cell line. DHM, dihydromyricetin; HCC, hepatocellular carcinoma.

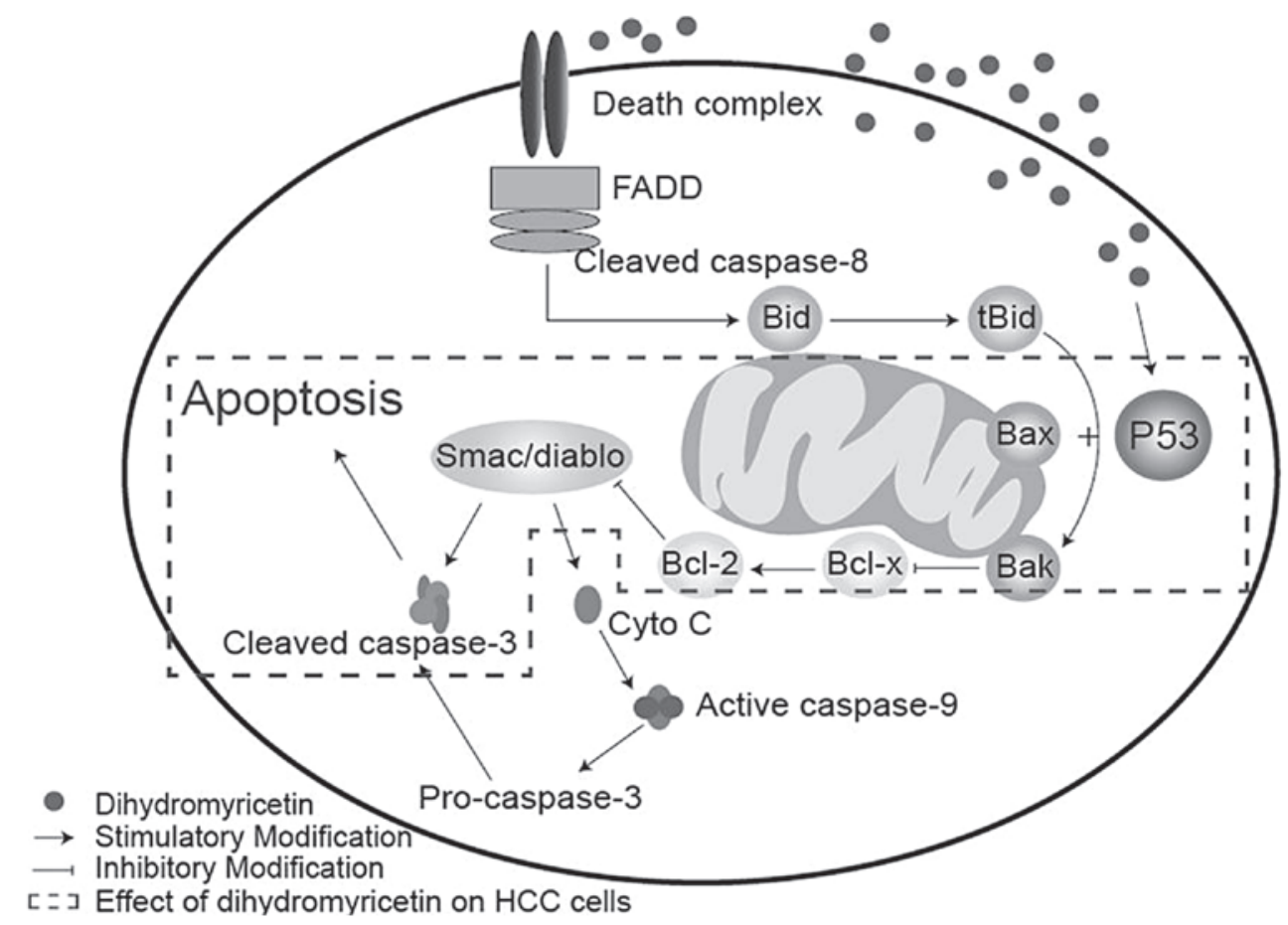

Figure 6. Potential mechanism of dihydromyricetin-induced cell apoptosis in HCC cells. HCC, hepatocellular carcinoma.

results demonstrated that DHM upregulates the levels of Bax, Bak, p53 and cleaved caspase-3 (p19) proteins in QGY7701, HepG2, MHcc97L and Hepal-6 cells. However, no significant differences in the expression of caspase-9 were identified. Furthermore, DHM did not affect the expression of p53, Bax, Bak or caspase-3 in HL7702 cells (Fig. 5).

\section{Discussion}

In the present study, DHM was found to significantly inhibit the proliferation of nine different types of HCC cell line when compared with two immortalized normal human hepatic cell lines, HL7702 and L-02. The cell cycle assay demonstrated that DHM did not induce cell cycle arrest in HCC cell lines or normal human hepatic cell lines. In addition, various concentrations of DHM did not induce apoptosis in HCC cells. Notably, DHM did not induce apoptosis in the normal human liver cell lines, which suggested that DHM may present a novel candidate for the treatment of HCC based on its selective effects on liver cancer cells rather than normal liver cells.

Cell apoptosis is a complex biological process linked with intricate pathways, whereby the activation of cysteine proteases (caspases) acts as a key intracellular regulator of cell apoptosis $(10,11)$. Specifically, caspase-3 is a key mediator in the caspase family (12). Caspase- 3 may be activated by a variety of activators, which are classified into two 
predominant pathways: The death receptor-mediated pathway, involving caspase- 8 and-10, and the mitochondrion-mediated pathway, involving caspase- $9(13,14)$. In the present study, no significant differences in caspase- 9 expression were identified following DHM treatment, which indicated that DHM selectively induces apoptosis in HCC cells directly via the death receptor-mediated pathway.

p53 is an important protein in the death receptor pathway, which is as an upstream regulator of pro-apoptotic Bax and Bak in mitochondria (15-17). Previous studies have demonstrated that p53 activates the transcription of Bax and Bak, whereby the activated Bax and Bak may coordinate with the release of cytochrome $c$ and Smac/diablo from the mitochondria, leading to the induction of caspase- 9 and -3 activation and/or cleavage, directly $(17,18)$. In the present study, p53, Bax and Bak were significantly upregulated following DHM treatment in different HCC cell lines; however, caspase-9 was not activated. The mechanism of DHM-induced cell apoptosis may occur as follows, the upregulation of p53 positively increases the expression of Bax/Bak, whilst simultaneously inhibiting $\mathrm{Bcl}-2$ protein. This then results in the activation of caspase-3, leading to cell apoptosis (Fig. 6). In addition, it was found that DHM did not affect the growth of immortalized normal human liver cells lines.

In conclusion, the results of the present study revealed that DHM effectively inhibits proliferation and induces apoptosis in HCC cells. In addition, DHM exhibited no significant hepatotoxicity to normal liver cells, which supports the possibility of DHM serving as a therapeutic candidate for HCC.

\section{Acknowledgements}

This study was supported by the National Natural Science Fund (grant no. 81041099) and the Guangdong Province Natural Science Fund (grant no. S2011010003750).

\section{References}

1. Chan SL and Yeo W: Targeted therapy of hepatocellular carcinoma present and future. J Gastroenterol Hepatol 27: 862-872, 2012.
2. Perz JF, Armstrong GL, Farrington LA, et al: The contributions of hepatitis $B$ virus and hepatitis $C$ virus infections to cirrhosis and primary liver cancer worldwide. J Hepatol 45: 529-538, 2006.

3. Takayama T, Sekine T, Makuuchi M, et al: Adoptive immunotherapy to lower postsurgical recurrence rates of hepatocellular carcinoma: a randomised trial. Lancet 356: 802-807, 2000.

4. Hu Y, Wang S, Wu X, et al: Chinese herbal medicine-derived compounds for cancer therapy: A focus on hepatocellular carcinoma. J Ethnopharmacol 149: 601-612, 2013.

5. Chen YQ, Ni DJ, Cheng Q, et al: Study on the hypolipidemic effect of flavones and dihydromyricetin From Tengcha. J Tea Sci 3: 221-225, 242, 2007

6. Zhong ZX, Zhou GF, Chen XF and Qin JP: Experimental study on the protective effect of dihydromyricetin from Guangxi Ampelopsis grossepentata on liver. Chin J Tradit Med Sci Technol 9: 155-156, 2002.

7. Xu JJ, Yao MJ and Wu MC: Study on biological efficacy of dihydromyricetin. Food Sci 29: 622-625, 2008.

8. Shen Y, Lindemeyer AK, Gonzalez C, et al: Dihydromyricetin as a novel anti-alcohol intoxication medication. J Neurosci 32 390-401, 2012

9. He GX, Yang WL, Pei G, et al: Studies on the effect of dihydromyricetin on antilipid-peroxidation. Zhongguo Zhong Yao Za Zhi 28: 1188-1190, 2003 (In Chinese).

10. Mu R, Lu N, Wang J, et al: An oxidative analogue of gambogic acid-induced apoptosis of human hepatocellular carcinoma cell line HepG2 is involved in its anticancer activity in vitro. Eur J Cancer Prev 19: 61-67, 2010.

11. Alenzi FQ, Alenazi BQ, AL-Anazy FH, et al: The role of caspase activation and mitochondrial depolarisation in cultured human apoptotic eosinophils. Saudi J Biol Sci 17: 29-36, 2010.

12. Wolf BB, Schuler M, Echeverri F and Green DR: Caspase-3 is the primary activator of apoptotic DNA fragmentation via DNA fragmentation factor-45/inhibitor of caspase-activated DNase inactivation. J Biol Chem 274: 30651-30656, 1999.

13. Fan TJ, Han LH, Cong RS and Liang J: Caspase family proteases and apoptosis. Acta Biochim Biophys Sin (Shanghai) 37: 719-727, 2005.

14. Ling Y, Lu N, Gao Y, et al: Endostar induces apoptotic effects in HUVECs through activation of caspase-3 and decrease of Bcl-2. Anticancer Res 29: 411-417, 2009.

15. Cheng EHY, Wei MC, Weiler S, et al: BCL-2, BCL-X(L) sequester $\mathrm{BH} 3$ domain-only molecules preventing BAX-and BAK-mediated mitochondrial apoptosis. Mol Cell 8: 705-711, 2001.

16. Hussein MR: Analysis of p53, BCL-2 and epidermal growth factor receptor protein expression in the partial and complete hydatidiform moles. Exp Mol Pathol 87: 63-69, 2009.

17. Degenhardt K, Chen G, Lindsten T and White E: BAX and BAK mediate p53-independent suppression of tumorigenesis. Cancer Cell 2: 193-203, 2002.

18. Henry H, Thomas A, Shen Y and White E: Regulation of the mitochondrial checkpoint in p53-mediated apoptosis confers resistance to cell death. Oncogene 21: 748-760, 2002. 